\title{
Relação entre a Funcionalidade e a Capacidade Motora de Pacientes Pós-AVC na Fase Aguda
}

\author{
Relationship between functionality and motor capacity of \\ acute stroke patients
}

\author{
Relación entre funcionalidad y capacidad motora en \\ pacientes después del AVC en la fase aguda
}

\author{
Tamires Cristine Bitencourt ${ }^{1}$, Fabiane Maria Klitzke dos Santos², \\ Antonio Vinicius Soares ${ }^{3}$
}

1.Fisioterapeuta, Residente do Programa Multiprofissional em Neurologia do Hospital São José - UNIVILLE, Joinville-SC, Brasil. ORCID: 0000-0002-4335-1463.

2.Fisioterapeuta, Especialista em Fisioterapia Neurofuncional no Adulto e Idoso, Hospital São José, Joinville-SC, Brasil. ORCID: 0000-0001-7603-7019.

3.Fisioterapeuta, Doutor em Ciências do Movimento Humano, Universidade da Região de Joinville e Faculdade IELUSC, Joinville-SC, Brasil, ORCID: 0000-0001-6090-1423.

\begin{abstract}
Resumo
Introdução. O AVC é a primeira causa de morte e incapacidade no Brasil e é de extrema importância a avaliação funcional e a caracterização desse paciente na fase aguda para avaliar o nível das limitações e dependências. Objetivo. Analisar a relação entre a independência funcional e a capacidade motora de pacientes hemiparéticos pós-AVC na fase aguda. Método. Estudo Descritivo Correlacional envolvendo 24 pacientes hemiparéticos nos primeiros dez dias da admissão hospitalar. Foram avaliados utilizando a Escala de Movimento da Mão (EMM), Mensuração da Força de Preensão Manual (FPM) por dinamometria, Teste de Controle de Tronco (TCT), Timed Up and Go (TUG), Teste de Velocidade da Marcha (TVM), Índice de Barthel (IB) e a Escala de Qualidade de vida específica para AVE (EQVE-AVE). A análise estatística foi descritiva e com o Teste de Correlação de Spearman, nível de significância de $1 \%(p<0,01)$. Resultados. A independência funcional demonstrou estar fortemente correlacionada com 0 controle de tronco, mobilidade funcional, marcha e força de preensão manual no lado afetado. Conclusões. Sugere-se que na fase aguda após o AVC a mobilidade funcional, o controle de tronco, a velocidade da marcha e a força de preensão manual no lado parético afetam diretamente a independência funcional destes indivíduos.
\end{abstract}

Unitermos. Acidente vascular cerebral; Paresia; Avaliação; Reabilitação Neurológica

\begin{abstract}
Introduction. Stroke is the leading cause of death and disability in Brazil and it is importance that functional assessment and characterization of this patient in the acute phase is to assess the level of limitations and dependence of this patient. Objective. To analyze the relationship between functional independence and motor capacity of post-stroke hemiparetic patients in the acute phase. Method. Correlational Descriptive Study involving 24 hemiparetic patients in the first ten days of hospital admission. They were evaluated by the following instruments: Hand Movement Scale (HMS), Hand Grip Force Measurement (HGF) by Dynamometry, Trunk Control Test (TCT), Timed Up and Go (TUG), Gait Speed Test (GST), Barthel Index (BI) and the Stroke Specific Quality of Life Scale (SSQLS). For the Statistical analysis we used the Spearman Correlation Test with a significance level of $1 \%(p<0.01)$ and a descriptive analysis. Results. Functional independence demonstrated to be strongly correlated with trunk control, functional mobility, gait, and handgrip strength on the affected side. Conclusions. It is suggested that in the acute phase after a stroke, functional mobility, trunk control, gait speed and handgrip strength on the paretic side directly affect the functional independence of these individuals.
\end{abstract}

Keywords. Stroke; Paresis; Assessment; Neurological Rehabilitation 


\section{Resumen}

Introducción. El AVC es la principal causa de muerte y discapacidad en Brasil y es de suma importancia que la evaluación funcional y la caracterización de este paciente en la fase aguda para evaluar el nivel de limitaciones y dependencia de este paciente. Objetivo. analizar la relación entre la independencia funcional y la capacidad motora de los pacientes hemiparéticos post accidente cerebrovascular en la fase aguda. Método. Estudio descriptivo correlacional con 24 pacientes hemipareticos en los primeros diez días de ingreso hospitalario. Se evaluaron mediante la Escala de movimiento manual (EMM), Medición de la fuerza de agarre manual (FPM) por dinamometría, Prueba de control del tronco (TCT), Timed Up and Go (TUG), Prueba de velocidad de la marcha (TVM), Índice de Barthel (IB) y la escala de calidad de vida específica para el AVE (EQVE-AVE). El análisis estadístico fue descriptivo y con la prueba de correlación de Spearman, un nivel de significación del $1 \%(p<0,01)$. Resultados. Se ha demostrado que la independencia funcional está fuertemente correlacionada con el control del tronco, la movilidad funcional, la marcha y la fuerza del mango en el lado afectado. Conclusiones. se sugiere que en la fase aguda después de un AVC, la movilidad funcional, el control del tronco, la velocidade de la marcha y la fuerza de agarre en el lado parético afectan directamente la independencia funcional de estos individuos.

Palabras clave. Accidente Cerebrovascular; Paresia; Evaluación; Rehabilitación Neurológica

Trabalho realizado no Hospital São José, Joinville-SC, Brasil.

Endereço para correspondência: Tamires C Bitencourt, R Luiz Maiochi, 250. Parque Esperança, Poços de Caldas-MG. CEP 37713-162. E-mail: miresb2@hotmail.com

\section{INTRODUÇÃO}

O Acidente Vascular Cerebral (AVC) é uma disfunção neurológica aguda de origem vascular, sendo uma doença grave, comum e de grande impacto na sociedade, capaz de originar sequelas que podem acometer as habilidades físicas como controle motor, equilíbrio, força e mobilidade, que costumam requerer tratamento de longo prazo ${ }^{1,2}$.

O AVC é a primeira causa de morte e incapacidade no Brasil. Assim, muitos sobreviventes experimentam dificuldade persistente para executar as tarefas diárias como uma consequência direta da doença, necessitando de auxílios físicos, sociais e emocionais ${ }^{1}$. 
Tendo em vista a diversidade de sequelas que podem surgir após o evento do AVC e considerando o aumento do número de sobreviventes com dependência física e dificuldades para realizar as atividades de vida diária $\left(\right.$ AVD's $^{3}$, torna-se imprescindível lançar mão de ferramentas que auxiliem no processo de qualificação e quantificação da nova condição de saúde do indivíduo, nas diferentes etapas do seu curso evolutivo ${ }^{4}$.

Dentre os distúrbios de movimentos secundários à lesão provocada pelo AVC a hemiparesia é a mais comumente encontrada, e se refere a uma fraqueza muscular no hemicorpo contralateral a lesão cerebral. Frequentemente esta fraqueza torna-se o gatilho para o surgimento de alterações posturais capazes de ocasionar déficits de equilíbrio e das habilidades motoras. Isso, por sua vez, dificulta diversas atividades funcionais rotineiras à vida e promove maior risco de queda ${ }^{5,6}$.

A funcionalidade e a incapacidade estão relacionadas com as condições de saúde do indivíduo, tendo em vista o que este indivíduo consegue ou não realizar, e são determinadas através do contexto ambiental em que eles vivem $^{1}$.

Sabe-se que após um evento agudo deve-se aproveitar os mecanismos de plasticidade cerebral para a melhor recuperação possível. Para isso, é importante prever e estratificar a recuperação funcional, desde os estágios iniciais até o potencial de recuperação funcional individual de longo prazo 4 . 
Com isso, a avaliação funcional e a caracterização do paciente pós-AVC na fase aguda são de extrema importância para avaliar o nível das limitações e dependência desses indivíduos ${ }^{4}$, sendo o Índice de Barthel um dos instrumentos mais utilizados para avaliar a funcionalidade de indivíduos pós AVC?.

Diante disso, o objetivo deste estudo foi analisar a relação entre a independência funcional e a capacidade motora de pacientes hemiparéticos pós-AVC na fase aguda.

\section{MÉTODO}

Trata-se de um estudo Descritivo Correlacional desenvolvido na Unidade de AVC Integral do Hospital São José (HSJ) do munícipio de Joinville - SC, durante os meses de Maio a Outubro de 2019. Esta pesquisa foi aprovada pelo Comitê de Ética em Pesquisa (CAAE:08261119.4.0000.5362).

\section{Amostra}

Foram incluídos no estudo indivíduos com diagnóstico clínico de primeiro episódio de AVC, com até 10 dias de internação e sequela de hemiparesia, maiores de 18 anos, sem distinção de gênero, raça e nível socioeconômico, e que após esclarecimentos e orientações assinaram o Termo de Consentimento Livre e Esclarecido. Foram excluídos do estudo os indivíduos que apresentavam déficits visuais e/ou 
auditivos graves e incapacidade de compreensão da realização dos testes.

A seleção dos pacientes foi realizada através da busca pelo sistema informatizado do hospital, após a internação na Unidade de AVC. Uma ficha cadastral foi utilizada para anamnese e coleta de dados sociodemográficos.

\section{Procedimento}

Para avaliação da capacidade motora foram utilizados os instrumentos descritos a seguir:

A funcionalidade da mão foi avaliada bilateralmente através da Escala de Movimentos da Mão (EMM), solicitandose que o indivíduo realizasse os seguintes movimentos: flexão ativa dos dedos em sinergia; flexão e extensão ativas de todos os dedos em sinergia; extensão do dedo indicador enquanto mantém os outros dedos em flexão; oposição do polegar ao dedo indicador; e oposição do polegar a todos os outros dedos, utilizando-se da pontuação de 1 a $6^{8}$.

A força de preensão palmar (FPP) foi mensurada no lado parético e não parético com o dinamômetro Smedley Saehan ${ }^{\circledR}$ (capacidade de mensuração até 100 kgf; sensibilidade de $0,1 \mathrm{kgf}$ ). O indivíduo foi avaliado na posição sentada com os quadris e joelhos a 900, antebraço e punho em posição neutra, enquanto o examinador sustentava o dinamômetro. O valor utilizado de cada mão foi a média de três medidas ${ }^{9}$.

O controle de tronco foi avaliado através do Teste de controle de Tronco (TCT) sendo solicitado ao paciente para 
executar quatro tarefas: rolar na cama para ambos os lados; passar da posição de decúbito dorsal para sentado; e manter o equilíbrio na posição sentada sem apoio dos pés no chão por mais de 30 segundos. A pontuação em cada item é atribuída de acordo com os seguintes critérios: 0 quando 0 indivíduo é incapaz de realizar a tarefa; 12 quando é capaz de realizar a tarefa, mas com dificuldade; e 25 quando é capaz de realizar a tarefa de forma adequada, totalizando 100 pontos $^{10}$.

A mobilidade funcional foi avaliada através do Timed Up and Go (TUG). Foi solicitado que o indivíduo se levantasse de uma cadeira com encosto, porém sem braços, caminhasse 3 metros, virasse $180^{\circ}$, voltasse rumo a cadeira e se sentasse novamente. O tempo total despendido para concluir todo o percurso foi quantificado em segundos ${ }^{11}$.

O Teste de Velocidade de Marcha (TVM) verificou a velocidade da marcha em um percurso total de 8 metros: 2 metros destinados para a fase de aceleração e 2 para a de desaceleração; e 4 metros efetivamente cronometrados. Foi solicitado ao indivíduo para caminhar na sua velocidade usual, sendo permitida a utilização de dispositivos auxiliares de marcha ${ }^{12}$.

A independência funcional foi obtida através do Índice de Barthel (IB). É uma medida amplamente utilizada de incapacidade funcional, e demonstra ser uma ferramenta válida e confiável para avaliar a independência funcional em pacientes com AVC em qualquer fase da doença ${ }^{13}$. A pontuação foi obtida através de entrevista com o paciente 
e/ou cuidador, no qual se questionou itens relacionados à capacidade dos indivíduos em realizar 10 atividades de vida diária avaliada como dependente ou independente, 8 referentes a atividades pessoais e 2 referentes à mobilidade. A pontuação é de 0 a 100, sendo que quanto mais alta maior é o grau de independência ${ }^{14}$.

Como complemento nas avaliações foi identificado o impacto na qualidade de vida das limitações impostas pelo AVC em sua fase aguda. Foi avaliada através da Escala de Qualidade de vida específica para AVE (EQVE-AVE) um questionário que compreende 49 itens, subdivididos em 12 dimensões: energia, papéis familiares, linguagem, mobilidade, humor, personalidade, autocuidado, papéis sociais, pensamento e memória, função do membro superior, visão e trabalho/produtividade. Em cada item pode haver até três possibilidades de resposta que apresentam pontuação de 1 a 5: grau de concordância com as afirmações expostas; grau de dificuldade vivido em relação às situações expostas; e grau de ajuda para executar as tarefas expostas. O escore mínimo da escala é de 49 pontos e o máximo de 245 pontos, onde se entende que uma pontuação igual ou menor a 147 configura um índice de qualidade de vida inferior e maior que 147 um índice de qualidade de vida superior ${ }^{15,16}$.

\section{Análise dos dados}

A tabulação e análise dos dados foram realizadas no

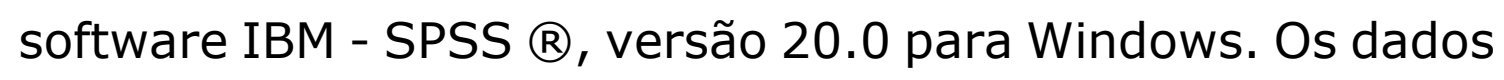
foram verificados quanto à normalidade e homogeneidade 
previamente respectivamente, pelo teste Shapiro-Wilk e teste de Levene. Foram obtidos dados de estatística descritiva como distribuição de frequência, média e desviopadrão. Para verificar a relação entre o IB e as demais variáveis do estudo foi utilizado o Teste de Correlação de Spearman com nível de significância de $1 \%(p<0,01)$.

\section{RESULTADOS}

Inicialmente foram analisados 212 prontuários, sendo 188 excluídos, 89 por não terem hemiparesia, 62 terem AVC prévio, 8 por alteração cognitiva grave, 9 por se recusarem a participar da pesquisa e $20 \mathrm{com}$ mais de dez dias do ictus devido a permanência na Unidade Terapia Intensiva ou na Unidade de AVC Agudo. Assim, 24 indivíduos elegíveis foram avaliados, sendo 12 homens e 12 mulheres, com média de

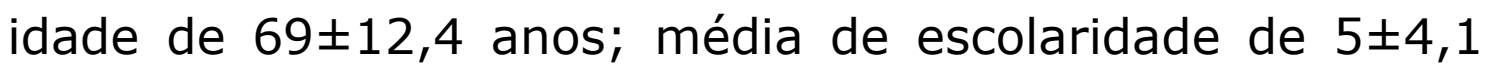
anos; e 54\% com hemiparesia à esquerda.

A Tabela 1 descreve as características clínicas dos participantes em relação à EMM, FPM, TCT, TUG, TVM, IB e EQVE-AVE.

As correlações entre o IB e as variáveis clínicas podem ser observadas na Tabela 2.

As análises de correlação revelaram que IB possui uma correlação moderada com o EQVE-AVE $(r=0,545 ; p=0,003)$ e com a $\operatorname{EMM~}(r=0,566 ; p=0,002)$, enquanto possui correlação muito forte com o TCT $(r=0,828 ; p=0,000)$. 
Tabela 1. Características clínicas dos indivíduos.

\begin{tabular}{|c|c|c|}
\hline Instrumentos de medida & $\mathbf{n}$ & Média (DP) \\
\hline Escala de Movimentos da Mão & 24 & $4,2(2,2)$ \\
\hline Força de Preensão Manual & 24 & \\
\hline Hemiparesia Direita & 11 & \\
\hline Lado parético & & $11,4(3,5)$ \\
\hline Lado não parético & & $24,9(2,6)$ \\
\hline Hemiparesia Esquerda & 13 & \\
\hline Lado parético & & $5,0(1,6)$ \\
\hline Lado não parético & & $15,8(2,4)$ \\
\hline Teste de Controle de Tronco & 24 & $68,6(29,9)$ \\
\hline Timed Up and Go & 12 & $24,3(18,5)$ \\
\hline Velocidade de Marcha & 12 & $11,5(8,9)$ \\
\hline & \multicolumn{2}{|c|}{ Distribuição de frequência (\%) } \\
\hline Índice de Barthel & 24 & \\
\hline$\leq 40$ dependência severa & 15 & 62,5 \\
\hline 41-59 dependência moderada & 1 & 4,2 \\
\hline 60-99 independência assistida & 7 & 29,2 \\
\hline 100 independência total & 1 & 4,2 \\
\hline $\begin{array}{l}\text { Escala de Qualidade de vida específica } \\
\text { para AVE }\end{array}$ & 24 & \\
\hline$\leq 147$ baixa qualidade de vida & 7 & 29,1 \\
\hline$>147$ boa qualidade de vida & 17 & 70,8 \\
\hline
\end{tabular}

$\mathrm{n}=$ número da amostra; DP: Desvio Padrão

Tabela 2. Correlação entre o Índice de Barthel e variáveis selecionadas.

\begin{tabular}{|c|c|c|c|c|c|c|}
\hline & TUG & TVM & TCT & FPMp & EMM & EQVE-AVE \\
\hline $\mathbf{r}$ & $-0,891$ & $-0,823$ & 0,828 & 0,640 & 0,566 & 0,545 \\
\hline Valor p & $0,000 *$ & $0,001 *$ & $0,000 *$ & $0,000 *$ & $0,002 *$ & $0,003 *$ \\
\hline $\mathbf{n}$ & 12 & 12 & 24 & 24 & 24 & 24 \\
\hline
\end{tabular}

*Nível de significância $\mathrm{p}=0,01 ; \mathrm{n}$ : número da amostra. IB, Índice de Barthel; TUG, Timed up and Go; EQVE-AVE, Escala de Qualidade de vida específica para AVE; TVM, Teste de Velocidade de Marcha; TCT, Teste de Controle de Tronco; FPMnp, Força de Preensão Manual do lado não parético; FPMp, Força de Preensão Manual do lado parético; EMM, Escala de Movimentos da Mão.

Foi encontrado ainda uma correlação forte do IB com a FPM do lado parético (FPMp) $(r=0,640, p=0,000)$. Porém, em contrapartida, não houve correlação significativa entre o IB 
e o teste de FPM do lado não parético (FPMnp) $(r=-0,026$, $\mathrm{p}=0,452)$.

Apenas 12 dos 24 participantes apresentaram condições físicas viáveis para a realização da avaliação com o TUG e o TVM. Os resultados obtidos a partir das correlações feitas do IB, tanto com o TUG $(r=-0,891, p=0,000)$, como com o TVM $(r=-0,823, p=0,001)$, apresentaram resultados muito fortes.

\section{DISCUSSÃO}

Nossos achados são semelhantes com literatura em relação a idade e escolaridade, entretanto não houve prevalência de gênero em nossa amostra. Sabe-se atualmente que a incidência do AVC aumenta com a idade, acometendo principalmente os idosos ${ }^{1,19}$, com baixa escolaridade ${ }^{17,18}$, e preferencialmente do sexo masculino ${ }^{17,19}$.

Cerca de $90 \%$ dos indivíduos acometidos pelo AVC possuem fatores de riscos modificáveis ${ }^{17}$. A baixa escolaridade influencia negativamente o conhecimento em relação aos fatores de risco, medidas preventivas e consequentemente 0 acesso aos cuidados da atenção primária ${ }^{18}$.

A hemiparesia é decorrente do acometimento da artéria cerebral média, é o quadro mais comumente encontrado após o $A \bigvee C^{5,6,20}$. Observou-se contradição com a literatura em nosso estudo, já que um dos principais motivos de exclusão foi a ausência deste comprometimento. 
Possivelmente, pela adoção de protocolos de neuroproteção adotados na Unidade de AVC. Além disso, a presença de AVC prévio representou uma parcela significativa dos indivíduos excluídos, indo ao encontro da literatura, no qual um terço dos pacientes que tiveram AVC está suscetível a outro evento $^{21}$. Isto reforça a necessidade de melhora da educação e do acesso à informação sobre os fatores de risco e consequentemente sua prevenção ${ }^{18,22}$.

Foi possível observar em nosso estudo que aproximadamente $96 \%$ das pessoas internadas na fase aguda do AVC apresentaram algum tipo de dependência. Considerando o resultado obtido pelo IB $62,5 \%$ possuíam dependência severa em relação à funcionalidade, repercutindo na necessidade de cuidados frequentes de familiares, pessoas próximas e da equipe de saúde.

Sabe-se que na fase aguda da doença, principalmente nas primeiras horas, toma-se um cuidado com a sua utilização, já que quase todas as pessoas durante este período recebem pontuações baixas no IB, seja devido ao déficit inicial, medicações, precaução médica ou risco de queda ${ }^{21}$.

Isso demonstra uma necessidade de se avaliar em qual momento utilizar o IB e ter uma visão qualitativa sobre ele, analisando o motivo pelo qual o paciente não consegue realizar as atividades, seja pelo déficit deixado pelo AVC ou por motivos técnicos não relacionados ao comprometimento que o impeça de completar a tarefa ${ }^{21}$. 
Apesar disso o IB é um teste rápido e confiável sendo o mais utilizado na prática clínica e em pesquisas para avaliar os prejuízos funcionais em pacientes pós-AVC ${ }^{3,23}$, além de ser um bom preditivo de funcionalidade e reabilitação $0^{3,21-25}$.

Pacientes avaliados dentro de uma semana após admissão hospitalar com escore maior que 60 pontos possuem uma tendência a permanecer menos tempo no hospital. Isso poderia estar relacionado ao fato deles conseguirem desempenhar de forma mais independente as atividades de vida diária no ambiente hospitalar, com exceção daquelas que envolvem higiene pessoal, banho e subir escadas, onde necessitam de algum auxílio ${ }^{21}$.

Pacientes que possuem uma pontuação de até 40 pontos no IB recebem alta hospitalar com pouca habilidade de mobilidade independente, menos de $50 \%$ possuem habilidades básicas como alimentar-se ${ }^{21}$. A maior parte dos participantes da pesquisa (62,5\%) não alcançou essa pontuação, o que poderia indicar um maior tempo de internação hospitalar, porém esse aspecto não foi abordado em nosso estudo.

O IB demonstrou estar correlacionado com vários instrumentos que avaliaram a capacidade motora dos indivíduos de nosso estudo. A correlação moderada entre o IB e a EMM demonstram que o déficit do membro superior do indivíduo, no âmbito hospitalar, não teve muita influência na sua funcionalidade, o que difere da literatura ${ }^{26}$. 0 resultado encontrado pode ser devido a pessoa não ter sido confrontada com os desafios diários extra hospitalares e/ou 
por não ter o entendimento da evolução da doença por estar na fase aguda ${ }^{26}$.

Em contrapartida houve uma forte correlação entre o IB e a dinamometria no lado parético, demonstrando que quanto menor a força de preensão do lado acometido, pior a funcionalidade do indivíduo. Esse resultado é compatível com outros estudos ${ }^{9,26,27}$, já que a utilização de ambas as mãos é comum nas atividades de vida diária. O prejuízo de uma delas afetará diretamente a otimização das tarefas e consequentemente a independência do indivíduo.

A correlação muito forte entre o IB e o TCT, demonstra que a habilidade de controle de tronco exerce influência significativa sobre o nível funcional do indivíduo na fase aguda. Isso é facilmente explicado, pois um bom controle de tronco é imprescindível para uma boa mobilidade e sua avaliação é utilizada como prognóstico de marcha. Uma pontuação maior que 50, como foi encontrada em nossa amostra, em até seis semanas após o AVC prediz uma recuperação da capacidade de andar em 18 semanas ${ }^{10}$.

Apenas 12 indivíduos conseguiram realizar os testes que necessitavam de deambulação, sendo eles o TUG e TVM. A média entre os pacientes que realizaram o TUG indicou mobilidade reduzida e alto risco de queda, considerando os valores de referência da literatura. Fica evidente a necessidade de cuidados maiores com pacientes pós-AVC na fase aguda ${ }^{11,28}$, já que $50 \%$ dos nossos indivíduos não conseguiram realizar o teste. 
Em relação a velocidade de marcha neste estudo, os indivíduos apresentaram um valor de $1,44 \mathrm{~m} / \mathrm{s}$, o que está acima daqueles encontrados na literatura, de $0,62 \mathrm{~m} / \mathrm{s}$ a $1,25 \mathrm{~m} / \mathrm{s}$, que seria a velocidade de marcha rápida para indivíduos hemiparéticos ${ }^{29}$. Considerando que essas pessoas já apresentam uma velocidade de marcha inferior quando comparado aos indivíduos saudáveis, esse aumento do tempo de realização do teste mostra risco ainda maior, sendo necessária a intensificação da proteção desses indivíduos ${ }^{29}$.

Além disso, a correlação forte do IB com TUG e com TVM reforça que as sequelas físicas afetam significativamente a independência funcional, quanto maior a sua incapacidade física pior a sua funcionalidade, o que corrobora com os dados encontrados na literatura 26,28,29.

Quando correlacionada a EQVE-AVE com o IB percebese uma correlação moderada. Aproximadamente $71 \%$ dos pacientes apresentaram boa qualidade de vida, o que diverge de alguns estudos que demonstram que as limitações físicas e o estresse psicológico influenciam na redução da qualidade de vida da pessoa e é um reflexo da incapacidade que acaba repercutindo em altos índices de dependência e sobrecarga familiar ${ }^{2,19}$.

Esse padrão positivo de percepção dos indivíduos internados sobre a sua própria qualidade de vida pode ser explicado pela tendência de atrelar as dificuldades assinaladas às restrições próprias do âmbito hospitalar e não necessariamente à sua nova condição física ${ }^{30}$, já que no momento da aplicação da escala eles ainda não tinham sido 
confrontados pela realidade em um cenário da rotina real, onde as habilidades são constantemente testadas.

Este estudo apresenta limitações, uma vez que o número de indivíduos da amostra é pequeno. Sugerem-se estudos com amostras maiores e seguimento dos indivíduos durante todo o período da internação hospitalar, a fim de observar melhor a influência das sequelas motoras sobre a independência funcional na fase aguda da doença e o quanto isto afeta o tempo de internação hospitalar. Desta forma poderemos compreender melhor as variáveis analisadas e o impacto da doença na vida do indivíduo. Além disso futuros desenhos de pesquisa poderiam contemplar aspectos cognitivos e emocionais, já que a ênfase nesta pesquisa, como detalhada no objetivo, tenha sido especialmente nos aspectos motores e funcionais.

\section{CONCLUSÃO}

Sugere-se que na fase aguda após o AVC a mobilidade funcional, o controle de tronco, a velocidade da marcha e a força de preensão manual no lado parético afetam diretamente $a$ independência funcional destes indivíduos. Outra informação extraída deste estudo foi a dificuldade de percepção dos pacientes sobre a sua nova condição física, considerando o momento agudo da doença, no qual não houve prejuízos na qualidade de vida. 


\section{REFERÊNCIAS}

1. Estrela TLRS, Assis SC, Daltro MCSL. Avaliação da funcionalidade de pacientes com sequelas de acidente vascular cerebral através da escala de Rankin. Fisioter Bras 2018;19:192-9. http://dx.doi.org/10.33233/fb.v19i5.2622

2. Marinho C, Monteiro M, Santos L, Oliveira-filho J, Pinto EB. Gait performance and quality of life in stroke survivors: a cross-sectional study. Rev Pesq Fisioterap 2018;8:79-87. http://dx.doi.org/10.17267/2238-2704rpf.v8i1.1777

3. Nave AH, Rackoll T, Grittner U, Bläsing H, Gorsler A, Nabavi DG, et al. Physical Fitness Training in Patients with Subacute Stroke (PHYSSTROKE): multicentre, randomised controlled, endpoint blinded trial. Rev Bras Atividade Fís e Saúde 2019;366:15101. http://dx.doi.org/10.1136/bmj.I5101

4.Lamola G, Pisa U, Bertolucci F, Rossi B, Chisari C. Clinical Assessments for Predicting Functional Recovery after Stroke. Int J Neurorehabil 2015;2:1-7. http://dx.doi.org/10.4172/23760281.1000174

5.Bambirra C, Cecília M, Rodrigues B, Danielli CMFC, Rodrigues PF. Clinical evaluation of balance in hemiparetic adults: a systematic review. Fisioter Mov 2015;28:187-200. http://dx.doi.org/10.1590/0103-5150.028.001.AR03

6. Monteiro J, Silva B. Efetividade da reabilitação virtual no equilíbrio corporal e habilidades motoras de indivíduos com déficit neuromotor: uma revisão sistemática. Rev Bras Atividade Fís Saúde 2012;17:2243. http://dx.doi.org/10.12820/rbafs.v.17n3p224-230

7. Musa KI, Keegan TJ. The change of Barthel Index scores from the time of discharge until 3-month postdischarge among acute stroke patients in Malaysia: A random intercept model. PloS One 2018;13:113. http://dx.doi.org/10.1371/journal.pone.0208594

8.Soares AV, Borges Junior NG, Domenech SC, Loch MSG. Deficits in upper limbs after stroke: influences of functional hemispheric asymmetries. Rev Neurocienc 2015;23:260-6. https://doi.org/10.4181/RNC.2015.23.02.992.7p

9.Soares AV, Kerscher C, Uhlig L, Domenech SC. Dinamometria de preensão manual como parâmetro de avaliação funcional do membro superior de pacientes hemiparéticos por acidente vascular cerebral. Fisioter Pesq 2011;18:359-64. http://dx.doi.org/10.1590/S180929502011000400011

10. Oliveira JS. Adaptação Cultural e Linguística do Trunk Control Test: avaliação das propriedades psicométricas (Dissertação). Portugal: Instituto Politécnico do Porto, 2015, 75p. https://recipp.ipp.pt/bitstream/10400.22/7863/1/DM JustinaOliveira 2015.pdf

11.Persson CU, Danielsson A, Sunnerhagen KS, Grimby-ekman A, Hansson P. Timed Up \& Go as a measure for longitudinal change in mobility after stroke - Postural Stroke Study in Gothenburg (POSTGOT). J Neuro Engin Rehabil 2014;11:1-7. http://dx.doi.org/10.1186/1743-0003-11-83 
12.Peters DM, Fritz SL, Krotish DE. Assessing the Reliability and Validity of a Shorter Walk Test Compared With the 10-Meter Walk Test for Measurements of Gait Speed in Healthy, Older Adults. Res Report 2013;26:24-30. http://dx.doi.org/10.1519/JPT.0b013e318248e20d

13.Caneda MAG, Fernandes JG, Almeida AG, Mugnol FE. Confiabilidade de escalas de comprometimento neurológico em pacientes com acidente vascular cerebral. Arq Neuropsiquiatr 2006;64:690-7. http://dx.doi.org/10.1590/S0004-282X2006000400034

14.Quinn TJ, Langhorne P, Stott DJ. Barthel Index for Stroke Trials. Stroke $2011 ; 42: 1146-51$.

http://dx.doi.org/10.1161/STROKEAHA.110.598540

15.Williams LS, Weinberger $M$, Harris LE, Clark DO, Biller J. Development of a stroke-specific quality of life scale. Stroke 1999;30:1362-9. http://dx.doi.org/10.1161/01.STR.30.7.1362

16. Lima RCM, Teixeira-Salmela LF, Magalhaes LC, Gomes-Neto M. Propriedades psicométricas da versão brasileira da escala de qualidade de vida específica para acidente vascular encefálico: aplicação do modelo Rasch. Rev Bras Fisioter 2008;12:149-56. http://dx.doi.org/10.1590/S1413-35552008000200012

17.Thrift AG, Cadilhac DA, Thayabaranathan T, Howard G, Howard VJ, Rothwell PM, et al. Global stroke statistics. Int J Stroke 2014;9:6-18. http://dx.doi.org/10.1177/1747493016676285

18. Medeiros CSP, Pacheco TBFP, Cavalcanti FAC, Cacho RO, Bezerra AMSB. Level of motor, sensory and functional impairment from stroke at admission and hospital discharge in Brazil. Brain Injury 2019;33:1430-5. http://dx.doi.org/10.1080/02699052.2019.1642515 19.Moreira RNTL, Andrade AS, Ribeiro KSQS, Nascimento J, Brito GEG. Qualidade de vida em indivíduos acometidos por Acidente Vascular Cerebral. Rev Neurocienc 2015;23:530-7.

https://doi.org/10.34024/rnc.2015.v23.7976

20.Maenza C, Good DC, Winstein CJ, Wagstaff DA, Sainburg RL. Functional Deficits in the Less-Impaired Arm of Stroke Survivors Depend on Hemisphere of Damage and Extent of Paretic Arm Impairment. Am Soc Neurorehabil Neural Repair 2019;34:39-50. http://dx.doi.org/10.1177/1545968319875951

21. Nakao S, Takata S, Uemura H, Kashihara M, Osawa T, Komatsu K, et al. Relationship between Barthel Index scores during the acute phase of rehabilitation and subsequent ADL in stroke patients. J Med Invest 2010;57:81-8. http://dx.doi.org/10.2152/jmi.57.81

22. Feigin VL, Krishnamurthi R, Bhattacharjee R, Parmar P, Theadom A, Hussein T, et al. New Strategy to Reduce the Global Burden of Stroke. Stroke $2015 ; 46: 1740-7$.

http://dx.doi.org/10.1161/STROKEAHA.115.008222

23.Schlemmer GBV, Machado AS, Santos TD, Tavares DI, Müller M, Pereira MB, et al. Correlação entre qualidade de vida e independência funcional de idosos institucionalizados. Rev Kairós Gerontol 2018;21:411-26. http://dx.doi.org/10.23925/2176901X.2018v21i1p411-426 
24.Iddagoda MT, Inderjeeth CA, Chan $\mathrm{K}$, Raymond WD. Prognostication accuracy of final destination in poststroke patients requiring transitional care. Australasian J Ageing 2019;00:1-7. http://dx.doi.org/10.1111/ajag.12742

25.Lee EH, Kim JW, Kang HJ, Kim SW, Kim JT, Park MS, et al. Association between Anxiety and Functional Outcomes in Patients with Stroke: A 1-Year Longitudinal Study. Psychiatry Investig 2017;1-21. http://dx.doi.org/10.30773/pi.2019.0188

26.Eraifej J, Clark W, France B, Desando S, Moore D. Effectiveness of upper limb functional electrical stimulation after stroke for the improvement of activities of daily living and motor function: a systematic review and meta-analysis. Syst Rev 2017;6:40. http://dx.doi.org/10.1186/s13643-017-0435-5

27.Silva SM, Carlos J, Corrêa F, Silva C Da, Fernanda P, Corrêa FI. Relação entre a força de preensão manual e capacidade funcional após Acidente Vascular Cerebral. Rev Neurocienc 2014;23:74-80. https://doi.org/10.34024/rnc.2015.v23.8052

28. Hafsteinsdottir TB, Rensink M, Schuurmans $M$. Clinimetric properties of the Timed Up and Go test for patients with stroke: a systematic review. Stroke Rehabilitation 2014;21:197-210. http://dx.doi.org/10.1310/tsr2103-197

29.Ricci NA, Ferrarias GP, Molina KI, Dib PM, Alouche SR. Velocidade de marcha e autoeficácia em quedas em indivíduos com hemiparesia após Acidente Vascular Encefálico. Fisioter Pesq 2015;22:191-6. http://dx.doi.org/10.590/1809-2950/14484522022015

30.Reis C, Faro A. Repercussões Psicológicas Após Um Acidente Vascular Cerebral (AVC): Uma Revisão De Literatura. Psicologia, Saúde \& Doenças 2019;20:16-32. http://dx.doi.org/10.15309/19psd200102 